\title{
Conformational Behavior of
}

\section{Tris(pentafluorophenyl)borane-Benzotriazole Adducts}

Francesca Focante,,$^{\dagger}$ Rino Leardini, ${ }^{\dagger}$ Andrea Mazzanti, ${ }^{* \dagger}$ Pierluigi Mercandelli, ${ }^{,}$and Daniele Nanni ${ }^{\dagger}$

† Dipartimento di Chimica Organica “A. Mangini”, Università di Bologna, viale Risorgimento 4, I-40136 Bologna (Italy) and ${ }^{\ddagger}$ Dipartimento di Chimica Strutturale e Stereochimica Inorganica, Università di Milano, via Venezian 21, I-20133 Milano (Italy).

mazzand@ms.fci.unibo.it

\section{SUPPORTING INFORMATION}

Page S2: $\quad$ Results for the homopolimerization of 1-hexene with adducts 1-3.

Page S3: $\quad$ ORTEP views of the $\mu-\left(1 H\right.$-Benzotriazolato- $\left.1 \kappa N^{1}: 2 \kappa N^{3}\right)$ diborate anion 3 .

Pages S4-S5: Selected geometric parameters for 3.

Pages S6-S10: Cartesian coordinates for the ab initio optimized structures of $\mathbf{1}$ and $\mathbf{3}$. 


\section{Results for the Homopolimerization of 1-Hexene with Adducts 1-3}

The homopolymerization of liquid 1-hexene was chosen as a simple screening tool to evaluate the performance of compounds 1-3 in alpha-olefin polymerization. The results are shown in Table S1. The choice of the metallocene catalyst selected for these experiments, that is dimethylsilyl $\{(1-(2,4,7-$ trimethylindenyl)-7-(2,5-dimethyl-cyclopenta[1,2-b:4,3-b']-dithiophene) $\}$ zirconium dimethyl, was based on its known ability to efficiently polymerize alpha-olefins to isotactic polyolefins., ${ }^{3,10}$

Although the results shown in Table S1 are preliminary and may be influenced by the purity of the various batches of 1-hexene used, nevertheless the indication is that in this process the efficency of adduct 3 is comparable to that of $\left[\mathrm{Ph}_{3} \mathrm{C}\right]\left[\mathrm{B}\left(\mathrm{C}_{6} \mathrm{~F}_{5}\right)_{4}\right]$ and $\left[\mathrm{HNMe}_{2} \mathrm{Ph}\right]\left[\mathrm{B}\left(\mathrm{C}_{6} \mathrm{~F}_{5}\right)_{4}\right]$, with adduct 3 giving higher molecular weights. Furthermore, 3 seems to be more effective even than methylalumoxane (MAO).

Table S1. 1-Hexene Polymerization Yields Using Borates or MAO as Cocatalysts. ${ }^{a}$

\begin{tabular}{|c|c|c|c|c|c|c|}
\hline Cocatalyst & $\mathrm{Mg}$ & $\begin{array}{l}\text { Yield } \\
(\mathrm{g})\end{array}$ & $\begin{array}{l}\text { Productivity } \\
\quad\left(\mathrm{kg} / \mathrm{g}_{\text {cat }}\right)\end{array}$ & $\begin{array}{l}\text { Productivity } \\
\left(\mathrm{kg} / \mathrm{g}_{\text {cocat }}\right)\end{array}$ & $\begin{array}{c}\mathrm{M}_{\mathrm{w}} \\
(\mathrm{kg} / \mathrm{mol})\end{array}$ & $\mathrm{M}_{\mathrm{w}} / \mathrm{M}_{\mathrm{n}}$ \\
\hline $\begin{array}{c}\mathrm{MAO}^{b} \\
(10 \% \mathrm{PhMe} \text { solution, } \mathrm{Al} / \mathrm{Zr}=200)\end{array}$ & 37.0 & 10.37 & 20.7 & 0.3 & 236.4 & 3.10 \\
\hline$\left[\mathrm{Ph}_{3} \mathrm{C}\right]\left[\mathrm{B}\left(\mathrm{C}_{6} \mathrm{~F}_{5}\right)_{4}\right]$ & 8.5 & 15.2 & 30.4 & 1.8 & 411.7 & 3.37 \\
\hline$\left[\mathrm{HNMe}_{2} \mathrm{Ph}\right]\left[\mathrm{B}\left(\mathrm{C}_{6} \mathrm{~F}_{5}\right)_{4}\right]$ & 7.4 & 14.4 & 28.8 & 1.9 & 400.3 & 3.83 \\
\hline $\begin{array}{c}\{\text { Benzotriazole- } \\
\left.\left[\mathrm{B}\left(\mathrm{C}_{6} \mathrm{~F}_{5}\right)_{3}\right]_{2}\right\}\left[\mathrm{NHEt}_{3}\right]^{b}\end{array}$ & 11.0 & 13 & 26.0 & 1.2 & 602.2 & 3.09 \\
\hline Benzotriazole-B $\left(\mathrm{C}_{6} \mathrm{~F}_{5}\right)_{3}$ & 5.8 & 0 & 0.0 & 0.0 & & \\
\hline$\left[\right.$ Benzotriazole- $\left.\mathrm{B}\left(\mathrm{C}_{6} \mathrm{~F}_{5}\right)_{3}\right]\left[\mathrm{HNEt}_{3}\right]^{b}$ & 6.7 & 0 & 0.0 & 0.0 & & \\
\hline$\left[\right.$ Benzotriazole- $\left.\mathrm{B}\left(\mathrm{C}_{6} \mathrm{~F}_{5}\right)_{3}\right]\left[\mathrm{HNEt}_{3}\right]^{c}$ & 6.7 & 0 & 0.0 & 0.0 & & \\
\hline $\begin{array}{l}{ }^{a} \text { Conditions: } 20 \mathrm{~g} \text { 1-hexene, } 50{ }^{\circ} \mathrm{C} \text {, } \\
\left.\text { clopenta[1,2-b:4,3-b- } b^{\prime} \text {-dithiophene) }\right\} \text { zirco } \\
\text { ercolated over activated alumina prior to } \\
\text { equence of addition: 1) 1-hexene }+\mathrm{Al}^{i} \mathrm{Bu}_{3} \\
\text { equence of addition: 1) 1-hexene }+\mathrm{Al}^{i} \mathrm{Bu}_{3} \text {, }\end{array}$ & $\mathrm{n}$ & Cat & alm & et & 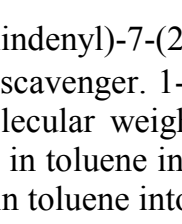 & 1 \\
\hline
\end{tabular}


Figure S1. ORTEP views of the $\mu-\left(1 H\right.$-benzotriazolato- $\left.1 \kappa N^{1}: 2 \kappa N^{3}\right)$ diborate anion 3 with a partial labeling scheme. Thermal ellipsoids are drawn at the $30 \%$ probability level; hydrogen atoms are given arbitrary radii. The two independent molecules are shown.

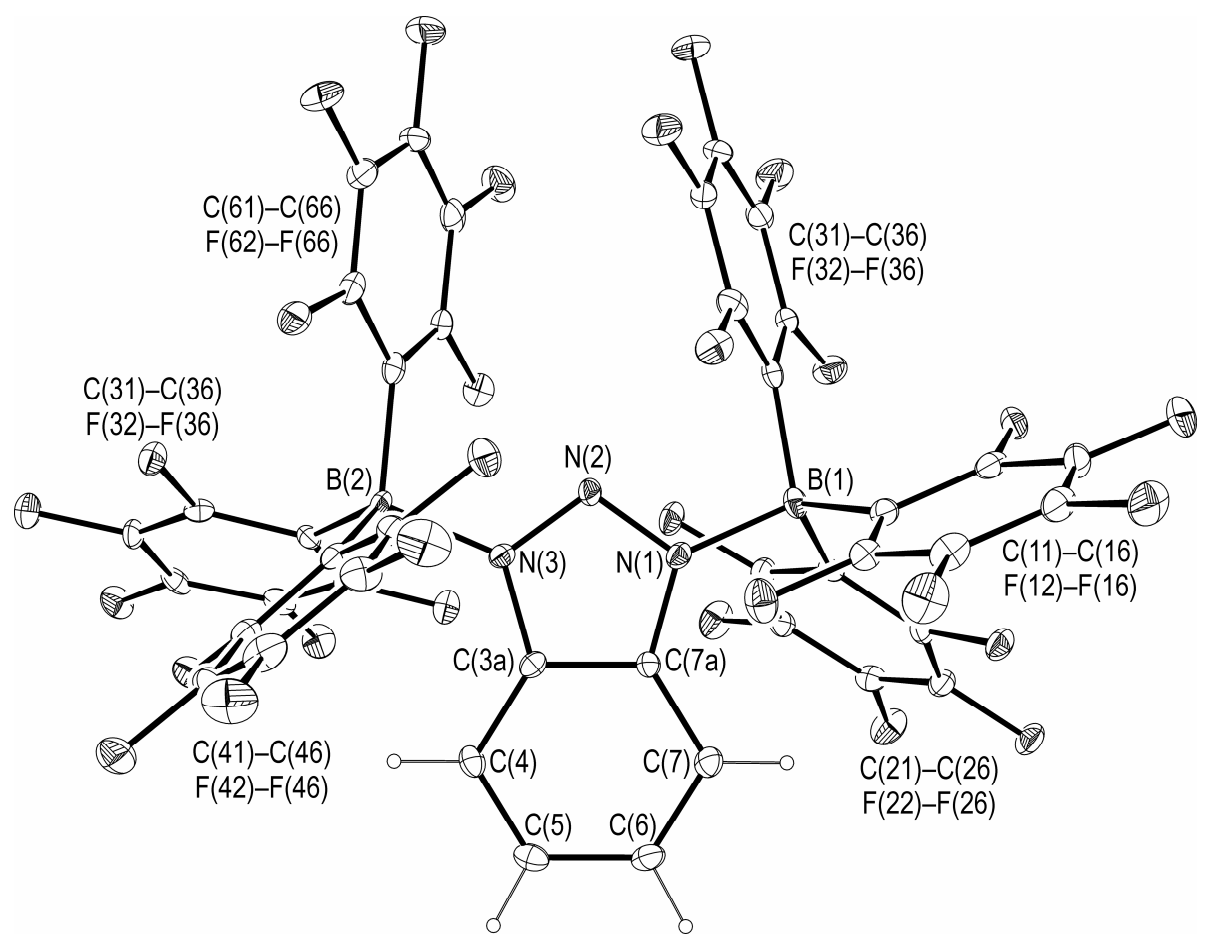

molecule I

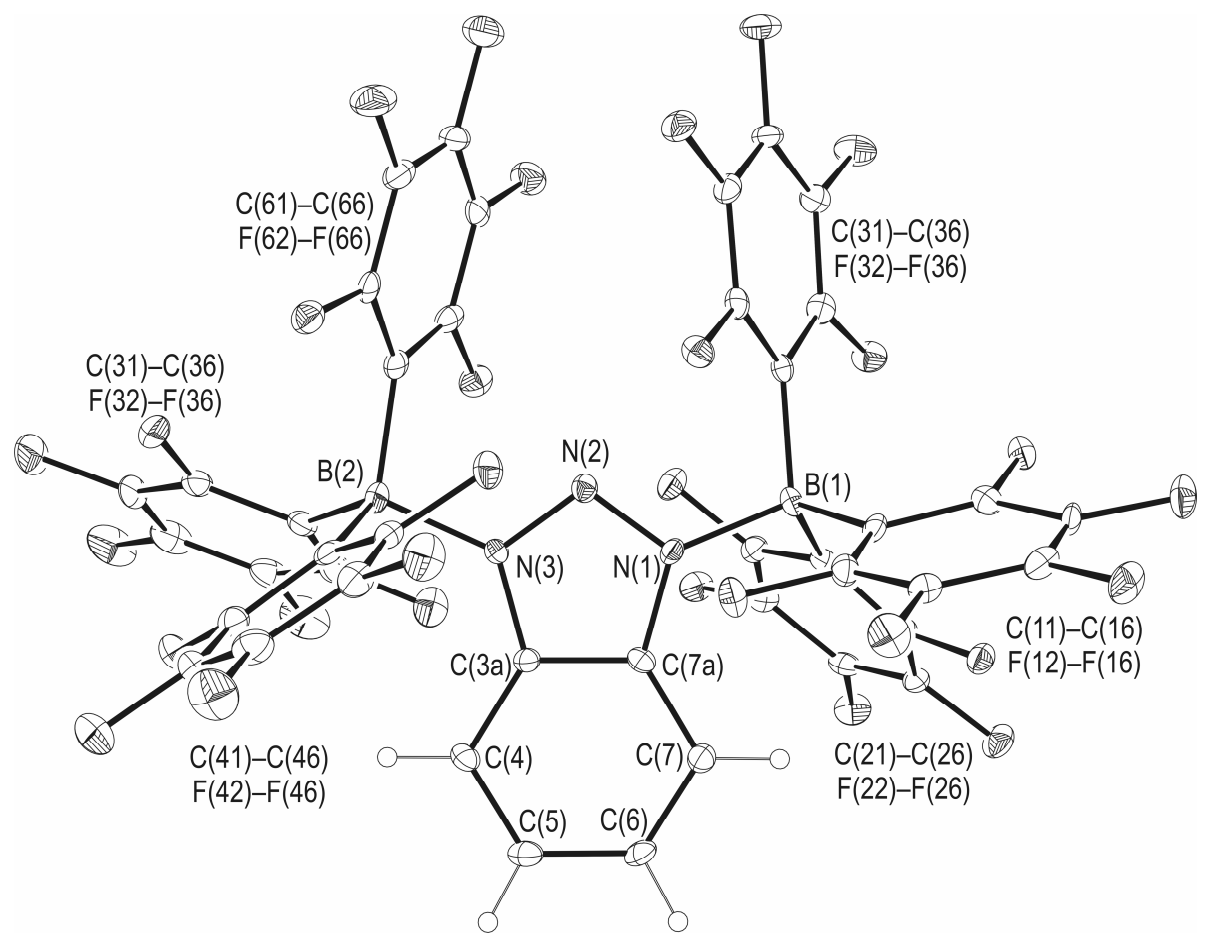

molecule II 
Table S2. Selected Geometric Parameters $\left(\AA\right.$ and $\left.^{\circ}\right)$ for $3{ }^{a}$

\begin{tabular}{|c|c|c|c|}
\hline & $\mathrm{I}$ & II & average $^{b}$ \\
\hline $\mathrm{N}(1)-\mathrm{N}(2)$ & $1.3254(18)$ & $1.3141(18)$ & \multirow{2}{*}{1.319} \\
\hline $\mathrm{N}(3)-\mathrm{N}(2)$ & $1.3176(18)$ & $1.3189(18)$ & \\
\hline $\mathrm{N}(1)-\mathrm{C}(7 \mathrm{a})$ & $1.376(2)$ & $1.3743(19)$ & \multirow{2}{*}{1.375} \\
\hline$N(3)-C(3 a)$ & $1.3740(19)$ & $1.375(2)$ & \\
\hline$C(3 a)-C(7 a)$ & $1.404(2)$ & $1.392(2)$ & 1.398 \\
\hline$C(3 a)-C(4)$ & $1.392(2)$ & $1.401(2)$ & \multirow{2}{*}{1.397} \\
\hline$C(7 a)-C(7)$ & $1.397(2)$ & $1.399(2)$ & \\
\hline$C(4)-C(5)$ & $1.371(2)$ & $1.382(2)$ & \multirow{2}{*}{1.375} \\
\hline$C(6)-C(7)$ & $1.372(2)$ & $1.374(2)$ & \\
\hline$C(5)-C(6)$ & $1.421(2)$ & $1.398(2)$ & 1.410 \\
\hline $\mathrm{N}(1)-\mathrm{B}(1)$ & $1.593(2)$ & $1.596(2)$ & \multirow{2}{*}{1.596} \\
\hline $\mathrm{N}(3)-\mathrm{B}(2)$ & $1.5996(19)$ & $1.596(2)$ & \\
\hline $\mathrm{B}(1)-\mathrm{C}(11)$ & $1.646(2)$ & $1.640(2)$ & \multirow{2}{*}{1.641} \\
\hline $\mathrm{B}(2)-\mathrm{C}(41)$ & $1.637(2)$ & $1.641(2)$ & \\
\hline $\mathrm{B}(1)-\mathrm{C}(21)$ & $1.633(2)$ & $1.641(2)$ & \multirow{2}{*}{1.640} \\
\hline $\mathrm{B}(2)-\mathrm{C}(51)$ & $1.635(2)$ & $1.650(3)$ & \\
\hline $\mathrm{B}(1)-\mathrm{C}(31)$ & $1.631(2)$ & $1.640(2)$ & \multirow{2}{*}{1.636} \\
\hline $\mathrm{B}(2)-\mathrm{C}(61)$ & $1.642(2)$ & $1.629(2)$ & \\
\hline $\mathrm{N}(2)-\mathrm{N}(1)-\mathrm{C}(7 \mathrm{a})$ & $109.75(12)$ & $110.05(12)$ & \multirow{2}{*}{109.92} \\
\hline $\mathrm{N}(2)-\mathrm{N}(3)-\mathrm{C}(3 \mathrm{a})$ & $109.93(12)$ & $109.95(12)$ & \\
\hline $\mathrm{N}(1)-\mathrm{N}(2)-\mathrm{N}(3)$ & $108.85(12)$ & $108.55(12)$ & 108.70 \\
\hline $\mathrm{N}(3)-\mathrm{C}(3 \mathrm{a})-\mathrm{C}(4)$ & $132.04(14)$ & $131.97(14)$ & \multirow{2}{*}{132.36} \\
\hline $\mathrm{N}(1)-\mathrm{C}(7 \mathrm{a})-\mathrm{C}(7)$ & $132.74(14)$ & $132.67(14)$ & \\
\hline $\mathrm{N}(3)-\mathrm{C}(3 \mathrm{a})-\mathrm{C}(7 \mathrm{a})$ & $105.87(13)$ & $105.67(13)$ & \multirow{2}{*}{105.73} \\
\hline $\mathrm{N}(1)-\mathrm{C}(7 \mathrm{a})-\mathrm{C}(3 \mathrm{a})$ & $105.59(13)$ & $105.78(13)$ & \\
\hline$C(4)-C(3 a)-C(7 a)$ & $122.01(14)$ & $122.36(14)$ & \multirow{2}{*}{121.90} \\
\hline$C(7)-C(7 a)-C(3 a)$ & $121.67(14)$ & $121.54(14)$ & \\
\hline $\mathrm{C}(3 \mathrm{a})-\mathrm{C}(4)-\mathrm{C}(5)$ & $116.25(14)$ & $115.64(14)$ & \multirow{2}{*}{115.86} \\
\hline$C(7 a)-C(7)-C(6)$ & $115.94(14)$ & $115.63(14)$ & \\
\hline$C(4)-C(5)-C(6)$ & $121.75(15)$ & $121.61(15)$ & \multirow{2}{*}{122.22} \\
\hline$C(7)-C(6)-C(5)$ & $122.32(15)$ & $123.21(15)$ & \\
\hline $\mathrm{N}(2)-\mathrm{N}(1)-\mathrm{B}(1)$ & $120.13(12)$ & $121.27(12)$ & \multirow{2}{*}{120.54} \\
\hline $\mathrm{N}(2)-\mathrm{N}(3)-\mathrm{B}(2)$ & $120.87(12)$ & $119.87(12)$ & \\
\hline $\mathrm{C}(7 \mathrm{a})-\mathrm{N}(1)-\mathrm{B}(1)$ & $129.14(12)$ & $128.17(12)$ & \multirow{2}{*}{128.90} \\
\hline $\mathrm{C}(3 \mathrm{a})-\mathrm{N}(3)-\mathrm{B}(2)$ & $128.82(12)$ & $129.46(13)$ & \\
\hline
\end{tabular}




\begin{tabular}{lccc}
\cline { 3 - 3 } $\mathrm{N}(1)-\mathrm{B}(1)-\mathrm{C}(11)$ & $111.51(12)$ & $110.24(12)$ & 111.29 \\
$\mathrm{~N}(3)-\mathrm{B}(2)-\mathrm{C}(41)$ & $111.11(12)$ & $112.29(14)$ & \\
$\mathrm{N}(1)-\mathrm{B}(1)-\mathrm{C}(21)$ & $103.05(12)$ & $101.00(11)$ & 102.02 \\
$\mathrm{~N}(3)-\mathrm{B}(2)-\mathrm{C}(51)$ & $101.97(11)$ & $102.08(12)$ & \\
$\mathrm{N}(1)-\mathrm{B}(1)-\mathrm{C}(31)$ & $105.60(12)$ & $108.97(12)$ & 107.04 \\
$\mathrm{~N}(3)-\mathrm{B}(2)-\mathrm{C}(61)$ & $107.61(12)$ & $105.96(12)$ & \\
$\mathrm{C}(11)-\mathrm{B}(1)-\mathrm{C}(21)$ & $114.33(13)$ & $115.86(13)$ & \\
$\mathrm{C}(41)-\mathrm{B}(2)-\mathrm{C}(51)$ & $114.13(12)$ & $114.58(13)$ & 114.72 \\
$\mathrm{C}(11)-\mathrm{B}(1)-\mathrm{C}(31)$ & $107.03(12)$ & $106.91(12)$ & \\
$\mathrm{C}(41)-\mathrm{B}(2)-\mathrm{C}(61)$ & $105.44(11)$ & $106.53(13)$ & 106.48 \\
$\mathrm{C}(21)-\mathrm{B}(1)-\mathrm{C}(31)$ & $115.01(13)$ & $113.65(12)$ & \\
$\mathrm{C}(51)-\mathrm{B}(2)-\mathrm{C}(61)$ & $116.47(12)$ & $115.15(14)$ & \\
$\mathrm{N}(2)-\mathrm{N}(1)-\mathrm{B}(1)-\mathrm{C}(11)$ & $115.19(16)$ & $119.86(15)$ & \\
$\mathrm{N}(2)-\mathrm{N}(3)-\mathrm{B}(2)-\mathrm{C}(41)$ & $121.34(15)$ & $120.59(16)$ & \\
$\mathrm{N}(1)-\mathrm{B}(1)-\mathrm{C}(11)-\mathrm{C}(12)$ & $-3.9(2)$ & $-11.5(2)$ & \\
$\mathrm{N}(3)-\mathrm{B}(2)-\mathrm{C}(41)-\mathrm{C}(42)$ & $-12.0(2)$ & $-9.3(3)$ & -9.2 \\
$\mathrm{~N}(1)-\mathrm{B}(1)-\mathrm{C}(21)-\mathrm{C}(22)$ & $54.72(19)$ & $54.79(18)$ & \\
$\mathrm{N}(3)-\mathrm{B}(2)-\mathrm{C}(51)-\mathrm{C}(52)$ & $61.20(17)$ & $59.2(2)$ & 57.5 \\
$\mathrm{~N}(1)-\mathrm{B}(1)-\mathrm{C}(31)-\mathrm{C}(32)$ & $62.66(19)$ & $57.7(2)$ & \\
$\mathrm{N}(3)-\mathrm{B}(2)-\mathrm{C}(61)-\mathrm{C}(62)$ & $59.2(2)$ & $58.3(2)$ & \\
\hline
\end{tabular}

${ }^{a} \mathrm{I}$ and II refer to the two independent molecules in the asymmetric unit of $\left[\mathrm{NHEt}_{3}\right] 3 \cdot{ }^{1 / 2} \mathrm{C}_{7} \mathrm{H}_{8} \cdot{ }^{b}$ Computed taking into account the idealized $C_{2}$ symmetry of anion 3 . 
Compound 1 - HF/6-31G optimized geometry

Energy calculated at HF/6-31G: -2590.32549456

\begin{tabular}{|c|c|c|c|}
\hline C & -2.74668800 & 1.96146800 & 3.95697600 \\
\hline $\mathrm{C}$ & -3.50264700 & 2.78847100 & 3.16463000 \\
\hline $\mathrm{C}$ & -3.30766300 & 2.86060700 & 1.76653400 \\
\hline C & -2.35689900 & 2.10512700 & 1.12825600 \\
\hline $\mathrm{C}$ & -1.59215100 & 1.25375800 & 1.93040700 \\
\hline $\mathrm{C}$ & -1.78316600 & 1.19537400 & 3.30003400 \\
\hline $\mathrm{N}$ & -0.85308000 & 0.28070100 & 3.72853800 \\
\hline $\mathrm{N}$ & -0.15171100 & -0.18711500 & 2.72842200 \\
\hline $\mathrm{N}$ & -0.55998000 & 0.36634000 & 1.65013600 \\
\hline $\mathrm{H}$ & -0.65684300 & -0.04565600 & 4.64389900 \\
\hline B & 0.02691000 & -0.05635900 & 0.18220200 \\
\hline $\mathrm{H}$ & -2.88640200 & 1.90999000 & 5.01750500 \\
\hline $\mathrm{H}$ & -4.25814100 & 3.40180300 & 3.61322800 \\
\hline $\mathrm{H}$ & -3.91754100 & 3.53024600 & 1.19444700 \\
\hline $\mathrm{H}$ & -2.20275400 & 2.17737500 & 0.07269200 \\
\hline $\mathrm{C}$ & -1.94627300 & -1.78186400 & -0.04033700 \\
\hline $\mathrm{C}$ & -1.33479900 & -0.64464900 & -0.55209000 \\
\hline $\mathrm{C}$ & -1.99752400 & -0.08944200 & -1.63192600 \\
\hline $\mathrm{C}$ & -3.13829300 & -0.62985600 & -2.18967900 \\
\hline $\mathrm{C}$ & -3.67994100 & -1.77237900 & -1.65921400 \\
\hline C & -3.07890400 & -2.35127600 & -0.56491000 \\
\hline$F$ & -1.40690000 & -2.38725500 & 1.06046000 \\
\hline $\mathrm{F}$ & -1.58056100 & 1.09470200 & -2.17527200 \\
\hline$F$ & -3.73837400 & -0.02142500 & -3.24244300 \\
\hline $\mathrm{F}$ & -4.80393100 & -2.30969000 & -2.18652100 \\
\hline$F$ & -3.61553800 & -3.46499900 & -0.00848400 \\
\hline $\mathrm{C}$ & 1.19403300 & 1.06903400 & -1.85722900 \\
\hline C & 0.73834400 & 1.23501700 & -0.55598600 \\
\hline $\mathrm{C}$ & 1.05655800 & 2.45727600 & 0.00732600 \\
\hline $\mathrm{C}$ & 1.74316300 & 3.44703600 & -0.66715800 \\
\hline $\mathrm{C}$ & 2.15623800 & 3.23439000 & -1.95693900 \\
\hline $\mathrm{C}$ & 1.88004200 & 2.02713100 & -2.55907100 \\
\hline$F$ & 0.95290600 & -0.11760200 & -2.48841100 \\
\hline $\mathrm{F}$ & 0.70215600 & 2.75355000 & 1.29376400 \\
\hline$F$ & 2.01258800 & 4.62770200 & -0.05577100 \\
\hline $\mathrm{F}$ & 2.83074800 & 4.19568700 & -2.62955500 \\
\hline$F$ & 2.28964800 & 1.79683700 & -3.83009300 \\
\hline $\mathrm{C}$ & 2.33000900 & -0.83008900 & 1.19838200 \\
\hline $\mathrm{C}$ & 1.23734500 & -1.15574400 & 0.40503200 \\
\hline C & 1.33956600 & -2.38869500 & -0.22005300 \\
\hline $\mathrm{C}$ & 2.41002200 & -3.24094500 & -0.04708900 \\
\hline $\mathrm{C}$ & 3.44994100 & -2.87794400 & 0.77108300 \\
\hline $\mathrm{C}$ & 3.40982000 & -1.65568900 & 1.39872900 \\
\hline $\mathrm{F}$ & 2.37121400 & 0.38684200 & 1.82069500 \\
\hline $\mathrm{F}$ & 0.36956300 & -2.82933100 & -1.06889200 \\
\hline $\mathrm{F}$ & 2.44132100 & -4.43697100 & -0.68433800 \\
\hline $\mathrm{F}$ & 4.50417700 & -3.70656100 & 0.95290100 \\
\hline $\mathrm{F}$ & 4.43317300 & -1.27127800 & 2.20232900 \\
\hline
\end{tabular}


Compound $3-C_{2}$ isomer, $\mathrm{HF} / 6-31 \mathrm{G}$ optimized geometry

Energy calculated at $\mathrm{HF} / 6-311+\mathrm{G}(\mathrm{d}):-4789.71701728$

\begin{tabular}{|c|c|c|c|}
\hline $\mathrm{N}$ & 0.32644500 & 1.01217200 & 0.78151800 \\
\hline $\mathrm{N}$ & 0.00000000 & 0.00000000 & 0.04023300 \\
\hline $\mathrm{N}$ & -0.32644500 & -1.01217200 & 0.78151800 \\
\hline $\mathrm{C}$ & -0.21027900 & -0.65826600 & 2.11261200 \\
\hline $\mathrm{C}$ & -0.41845200 & -1.36140000 & 3.30314200 \\
\hline $\mathrm{H}$ & -0.71716300 & -2.38801100 & 3.30892500 \\
\hline $\mathrm{C}$ & -0.20444900 & -0.67578600 & 4.47113500 \\
\hline $\mathrm{H}$ & -0.34963700 & -1.17444000 & 5.40900800 \\
\hline $\mathrm{C}$ & 0.20444900 & 0.67578600 & 4.47113500 \\
\hline $\mathrm{H}$ & 0.34963700 & 1.17444000 & 5.40900800 \\
\hline $\mathrm{C}$ & 0.41845200 & 1.36140000 & 3.30314200 \\
\hline $\mathrm{H}$ & 0.71716300 & 2.38801100 & 3.30892500 \\
\hline $\mathrm{C}$ & 0.21027900 & 0.65826600 & 2.11261200 \\
\hline B & 0.88103100 & 2.39083900 & 0.17637300 \\
\hline $\mathrm{C}$ & -0.10849100 & 3.66606000 & 0.55864800 \\
\hline $\mathrm{C}$ & -1.37444900 & 3.60932800 & 1.11598000 \\
\hline $\mathrm{C}$ & -2.16374400 & 4.72539000 & 1.31560000 \\
\hline $\mathrm{C}$ & -1.70867500 & 5.96299700 & 0.94228500 \\
\hline $\mathrm{C}$ & -0.46309200 & 6.07224600 & 0.36798700 \\
\hline C & 0.29394700 & 4.94260800 & 0.18827800 \\
\hline $\mathrm{C}$ & 2.40502300 & 2.47783100 & 0.83439800 \\
\hline C & 3.35353900 & 1.52761200 & 0.47342000 \\
\hline $\mathrm{C}$ & 4.63074200 & 1.48789500 & 0.97469200 \\
\hline $\mathrm{C}$ & 5.02140000 & 2.42207400 & 1.90632200 \\
\hline $\mathrm{C}$ & 4.11991500 & 3.36739800 & 2.31930300 \\
\hline $\mathrm{C}$ & 2.84639200 & 3.37641000 & 1.78795300 \\
\hline $\mathrm{C}$ & 0.84359600 & 2.31860500 & -1.47683000 \\
\hline $\mathrm{C}$ & -0.35917600 & 2.06188400 & -2.12384600 \\
\hline $\mathrm{C}$ & -0.50384500 & 2.02794200 & -3.49071800 \\
\hline $\mathrm{C}$ & 0.58222800 & 2.28413300 & -4.29307600 \\
\hline C & 1.78740600 & 2.57562600 & -3.70730900 \\
\hline $\mathrm{C}$ & 1.89835600 & 2.59447400 & -2.33287400 \\
\hline $\mathrm{F}$ & -1.92533800 & 2.42553200 & 1.50839900 \\
\hline$F$ & -3.39252500 & 4.60392000 & 1.88112500 \\
\hline $\mathrm{F}$ & -2.47452500 & 7.06646100 & 1.13317200 \\
\hline$F$ & 0.00000000 & 7.29069700 & -0.01416800 \\
\hline $\mathrm{F}$ & 1.52050700 & 5.10684700 & -0.39221300 \\
\hline$F$ & 3.02325200 & 0.57325000 & -0.43984400 \\
\hline$F$ & 5.51164300 & 0.54105800 & 0.56516900 \\
\hline$F$ & 6.27665600 & 2.39802100 & 2.41936900 \\
\hline$F$ & 4.47768100 & 4.28003100 & 3.26123500 \\
\hline $\mathrm{F}$ & 2.00090700 & 4.31690200 & 2.31619600 \\
\hline$F$ & -1.49076000 & 1.86209700 & -1.38933500 \\
\hline $\mathrm{F}$ & -1.71059600 & 1.76136800 & -4.05429300 \\
\hline$F$ & 0.45757500 & 2.26928900 & -5.64276200 \\
\hline $\mathrm{F}$ & 2.86308200 & 2.85856900 & -4.48629000 \\
\hline $\mathrm{F}$ & 3.12919800 & 2.93300100 & -1.85396600 \\
\hline B & -0.88103100 & -2.39083900 & 0.17637300 \\
\hline $\mathrm{C}$ & 0.10849100 & -3.66606000 & 0.55864800 \\
\hline $\mathrm{C}$ & 1.37444900 & -3.60932800 & 1.11598000 \\
\hline $\mathrm{C}$ & 2.16374400 & -4.72539000 & 1.31560000 \\
\hline $\mathrm{C}$ & 1.70867500 & -5.96299700 & 0.94228500 \\
\hline $\mathrm{C}$ & 0.46309200 & -6.07224600 & 0.36798700 \\
\hline $\mathrm{C}$ & -0.29394700 & -4.94260800 & 0.18827800 \\
\hline $\mathrm{C}$ & -2.40502300 & -2.47783100 & 0.83439800 \\
\hline $\mathrm{C}$ & -3.35353900 & -1.52761200 & 0.47342000 \\
\hline C & -4.63074200 & -1.48789500 & 0.97469200 \\
\hline $\mathrm{C}$ & -5.02140000 & -2.42207400 & 1.90632200 \\
\hline $\mathrm{C}$ & -4.11991500 & -3.36739800 & 2.31930300 \\
\hline $\mathrm{C}$ & -2.84639200 & -3.37641000 & 1.78795300 \\
\hline $\mathrm{C}$ & -0.84359600 & -2.31860500 & -1.47683000 \\
\hline
\end{tabular}




$\begin{array}{lrrr}C & 0.35917600 & -2.06188400 & -2.12384600 \\ C & 0.50384500 & -2.02794200 & -3.49071800 \\ C & -0.58222800 & -2.28413300 & -4.29307600 \\ C & -1.78740600 & -2.57562600 & -3.70730900 \\ C & -1.89835600 & -2.59447400 & -2.33287400 \\ F & 1.92533800 & -2.42553200 & 1.50839900 \\ F & 3.39252500 & -4.60392000 & 1.88112500 \\ F & 2.47452500 & -7.06646100 & 1.13317200 \\ F & 0.00000000 & -7.29069700 & -0.01416800 \\ F & -1.52050700 & -5.10684700 & -0.39221300 \\ F & -3.02325200 & -0.57325000 & -0.43984400 \\ F & -5.51164300 & -0.54105800 & 0.56516900 \\ F & -6.27665600 & -2.39802100 & 2.41936900 \\ F & -4.47768100 & -4.28003100 & 3.26123500 \\ F & -2.00090700 & -4.31690200 & 2.31619600 \\ F & 1.49076000 & -1.86209700 & -1.38933500 \\ F & 1.71059600 & -1.76136800 & -4.05429300 \\ F & -0.45757500 & -2.26928900 & -5.64276200 \\ F & -2.86308200 & -2.85856900 & -4.48629000 \\ F & -3.12919800 & -2.93300100 & -1.85396600\end{array}$


Compound $3-C_{s}$ isomer, $\mathrm{HF} / 6-31 \mathrm{G}$ optimized geometry

\section{Energy calculated at HF/6-311+G(d): -4789.71431984}

\begin{tabular}{|c|c|c|c|}
\hline $\mathrm{N}$ & -1.07978300 & 0.18582800 & -0.81969900 \\
\hline $\mathrm{N}$ & -0.00932700 & -0.15244500 & -0.16874400 \\
\hline $\mathrm{N}$ & 1.04775000 & 0.25843700 & -0.79555100 \\
\hline $\mathrm{C}$ & 0.66565800 & 0.92814000 & -1.94193400 \\
\hline $\mathrm{C}$ & 1.38922400 & 1.58523100 & -2.94200800 \\
\hline $\mathrm{H}$ & 2.45609500 & 1.65292700 & -2.91490300 \\
\hline $\mathrm{C}$ & 0.66574600 & 2.13568500 & -3.96767600 \\
\hline $\mathrm{H}$ & 1.17738000 & 2.64797000 & -4.75824800 \\
\hline $\mathrm{C}$ & -0.74176000 & 2.02918100 & -4.01984300 \\
\hline $\mathrm{H}$ & -1.26377500 & 2.45156800 & -4.85560700 \\
\hline $\mathrm{C}$ & -1.45030400 & 1.39238300 & -3.03434000 \\
\hline $\mathrm{H}$ & -2.51316000 & 1.29259100 & -3.09002500 \\
\hline $\mathrm{C}$ & -0.71499900 & 0.85736600 & -1.97140300 \\
\hline B & -2.55442400 & -0.01153400 & -0.20713800 \\
\hline $\mathrm{C}$ & -3.40248800 & -1.18472000 & -1.00733200 \\
\hline $\mathrm{C}$ & -2.96119600 & -1.96220600 & -2.06305000 \\
\hline $\mathrm{C}$ & -3.71447700 & -2.97674000 & -2.62094300 \\
\hline $\mathrm{C}$ & -4.96114500 & -3.25672900 & -2.12516900 \\
\hline $\mathrm{C}$ & -5.44601200 & -2.51348300 & -1.07366000 \\
\hline $\mathrm{C}$ & -4.67060300 & -1.51229600 & -0.54693700 \\
\hline $\mathrm{C}$ & -2.40078700 & -0.54523000 & 1.35543300 \\
\hline $\mathrm{C}$ & -2.91346400 & 0.06863200 & 2.48762500 \\
\hline $\mathrm{C}$ & -2.77975700 & -0.44401900 & 3.76068900 \\
\hline $\mathrm{C}$ & -2.11825500 & -1.62878300 & 3.95535900 \\
\hline $\mathrm{C}$ & -1.61108100 & -2.29263200 & 2.86477400 \\
\hline $\mathrm{C}$ & -1.76922600 & -1.75641200 & 1.60895200 \\
\hline $\mathrm{C}$ & -3.21886100 & 1.50994100 & -0.33198800 \\
\hline $\mathrm{C}$ & -4.35723000 & 1.86755200 & -1.03192900 \\
\hline $\mathrm{C}$ & -4.84399000 & 3.15840400 & -1.09378800 \\
\hline $\mathrm{C}$ & -4.18120400 & 4.16873900 & -0.44894000 \\
\hline $\mathrm{C}$ & -3.02979900 & 3.87488800 & 0.24475600 \\
\hline $\mathrm{C}$ & -2.57996000 & 2.57959700 & 0.28492700 \\
\hline$F$ & -1.73776500 & -1.75851900 & -2.62737600 \\
\hline$F$ & -3.22854800 & -3.69944200 & -3.66297500 \\
\hline$F$ & -5.71137100 & -4.24989900 & -2.66521400 \\
\hline$F$ & -6.68176500 & -2.77558100 & -0.57376400 \\
\hline$F$ & -5.20751900 & -0.79208100 & 0.48431300 \\
\hline$F$ & -3.61983900 & 1.23316000 & 2.40663000 \\
\hline$F$ & -3.30999300 & 0.21356200 & 4.82411400 \\
\hline$F$ & -1.97997100 & -2.14536600 & 5.20088500 \\
\hline $\mathrm{F}$ & -0.96256200 & -3.47457300 & 3.03594500 \\
\hline $\mathrm{F}$ & -1.28136900 & -2.49748100 & 0.57225000 \\
\hline$F$ & -5.05188400 & 0.95163900 & -1.77304900 \\
\hline$F$ & -5.96506000 & 3.43475600 & -1.81062500 \\
\hline$F$ & -4.64164200 & 5.44276800 & -0.50796100 \\
\hline$F$ & -2.35171200 & 4.86818000 & 0.87331400 \\
\hline$F$ & -1.43249800 & 2.35387700 & 0.98757500 \\
\hline B & 2.51688500 & 0.11115700 & -0.16150300 \\
\hline $\mathrm{C}$ & 3.60589700 & -0.55330700 & -1.22634300 \\
\hline $\mathrm{C}$ & 3.33205800 & -1.23186600 & -2.40246500 \\
\hline $\mathrm{C}$ & 4.30605000 & -1.84520500 & -3.16700800 \\
\hline $\mathrm{C}$ & 5.61567800 & -1.81444300 & -2.76532400 \\
\hline $\mathrm{C}$ & 5.93926600 & -1.16952900 & -1.59404400 \\
\hline $\mathrm{C}$ & 4.94672800 & -0.56934100 & -0.86161300 \\
\hline $\mathrm{C}$ & 2.84936300 & 1.67861100 & 0.27846500 \\
\hline $\mathrm{C}$ & 2.14983500 & 2.26418900 & 1.32766600 \\
\hline $\mathrm{C}$ & 2.32192300 & 3.56443100 & 1.73423300 \\
\hline $\mathrm{C}$ & 3.21777200 & 4.36904300 & 1.06865600 \\
\hline $\mathrm{C}$ & 3.91499600 & 3.85343900 & 0.00761500 \\
\hline $\mathrm{C}$ & 3.71873600 & 2.53997700 & -0.36574200 \\
\hline
\end{tabular}




$\begin{array}{lrrr}\text { C } & 2.47624600 & -0.98499600 & 1.07657200 \\ C & 1.99452100 & -2.26658100 & 0.83497400 \\ C & 1.99854500 & -3.27868000 & 1.76510600 \\ C & 2.51367800 & -3.04081700 & 3.01698400 \\ C & 3.02313800 & -1.79970500 & 3.30327300 \\ C & 3.00631700 & -0.80830700 & 2.34445700 \\ \text { F } & 2.06293600 & -1.33289400 & -2.88426900 \\ F & 3.97246200 & -2.48229100 & -4.31924600 \\ F & 6.58037300 & -2.41335600 & -3.50787500 \\ F & 7.23114600 & -1.13721300 & -1.17546900 \\ F & 5.32566000 & 0.04155400 & 0.30056500 \\ F & 1.23655100 & 1.52505800 & 2.01786500 \\ F & 1.62174300 & 4.06466100 & 2.78230200 \\ F & 3.39997600 & 5.65772100 & 1.44997000 \\ F & 4.78574200 & 4.64007600 & -0.67849300 \\ F & 4.42501000 & 2.13339300 & -1.46863900 \\ F & 1.52578600 & -2.58197100 & -0.40578700 \\ F & 1.53107800 & -4.51156300 & 1.45024800 \\ F & 2.54183300 & -4.02541000 & 3.94804100 \\ F & 3.55829200 & -1.55892100 & 4.52877700 \\ F & 3.57735600 & 0.37367400 & 2.71436100\end{array}$

